\title{
Impact Evaluation of Training Natural Leaders during a Community- Led Total Sanitation Intervention: A Cluster-Randomized Field Trial in Ghana
}

\author{
Jonny Crocker, ${ }^{*}{ }^{\dagger}$ Elvis Abodoo, ${ }^{\ddagger}$ Daniel Asamani, ${ }^{\ddagger}$ William Domapielle, ${ }^{\ddagger}$ Benedict Gyapong, ${ }^{\ddagger}$ \\ and Jamie Bartram ${ }^{\dagger}$ \\ ${ }^{\dagger}$ The Water Institute, University of North Carolina at Chapel Hill, Chapel Hill, North Carolina 27599-7431, United States \\ ${ }^{\ddagger}$ Plan International Ghana, No. 10 Yiyiwa Street, Abelemkpe, Accra, Ghana
}

Supporting Information

\begin{abstract}
We used a cluster-randomized field trial to evaluate training natural leaders (NLs) as an addition to a community-led total sanitation (CLTS) intervention in Ghana. NLs are motivated community members who influence their peers' behaviors during CLTS. The outcomes were latrine use and quality, which were assessed from surveys and direct observation. From October 2012, Plan International Ghana (Plan) implemented CLTS in 60 villages in three regions in Ghana. After 5 months, Plan trained eight NLs from a randomly selected half of the villages, then continued implementing CLTS in all villages for 12 more months. The NL training led to increased time spent on CLTS by community members, increased latrine construction, and a

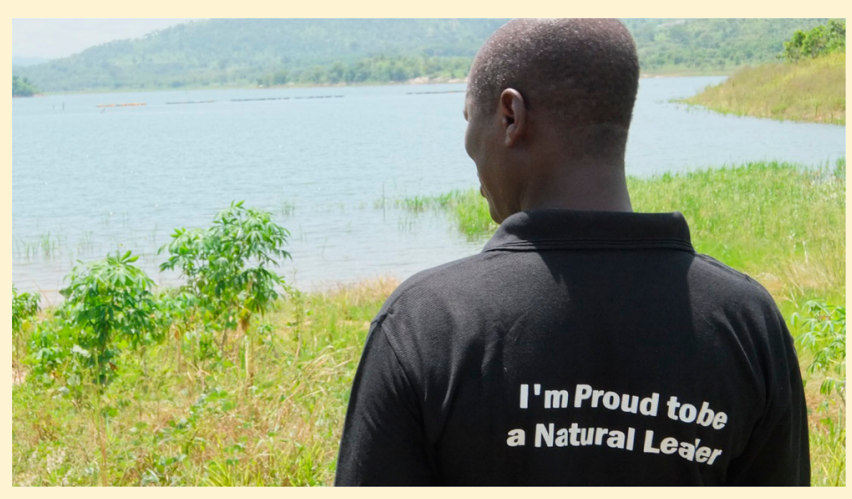
19.9 percentage point reduction in open defecation $(p<$ 0.001). The training had the largest impact in small, remote villages with low exposure to prior water and sanitation projects, and may be most effective in socially cohesive villages. For both interventions, latrines built during CLTS were less likely to be constructed of durable materials than pre-existing latrines, but were equally clean, and more often had handwashing materials. CLTS with NL training contributes to three parts of Goal 6 of the Sustainable Development Goals: eliminating open defecation, expanding capacity-building, and strengthening community participation.
\end{abstract}

\section{INTRODUCTION}

Globally, 2.4 billion people lack improved sanitation, and 946 million people practice open defecation. ${ }^{1}$ Fecal contamination of the environment from poor sanitation together with poor handwashing cause an estimated 577000 deaths annually. ${ }^{2}$ Reducing detrimental impacts of poor sanitation and hygiene requires both increased access and use of facilities.

A number of approaches have emerged for promoting sanitation and hygiene behavior change, ${ }^{3}$ including community-led total sanitation (CLTS). ${ }^{4}$ CLTS is a participatory approach in which an external facilitator triggers an awareness of sanitation and hygiene issues with the aim of generating collective action to eliminate open defecation (OD). ${ }^{4}$ CLTS facilitators encourage the most motivated community members - "natural leaders" - to lead their community by example, by building a latrine and convincing others to do likewise. There are a few impact evaluations of CLTS; one in Mali showed an impact on latrine use and child stunting, ${ }^{5}$ one in Tanzania showed an impact on latrine use but negligible impact on health outcomes, ${ }^{6}$ and one in Bangladesh did not show any impact. ${ }^{7}$ However, baseline open defecation was low in the Bangladesh study, which reduces the potential for impact and may reduce effectiveness. "Social capital" (community participation) may also influence CLTS effectiveness; another evaluation from Indonesia found that CLTS failed or even had adverse effects in villages with low social capital. ${ }^{9}$ An editorial on participatory development hypothesized that interventions in communities with "endogenous imperfections" (low social capital) may lead to appropriation of benefits by the most powerful. ${ }^{10}$

Diffusion theory can aid in understanding CLTS effectiveness. Diffusion theory describes factors that determine how a new technology or behavior (like latrine use) will spread through a community. ${ }^{11}$ Diffusion theory suggests that adoption of an innovation is initiated by external factors (like triggering), and that diffusion beyond early adopters depends on social networks and peer-communication. It also emphasizes that there are influential opinion leaders within communities. Natural leaders are mentioned frequently in the CLTS Handbook ${ }^{4}$ and in gray literature, ${ }^{12}$ and are described similarly to early adopters and

Received: March 30, 2016

Revised: July 15, 2016

Accepted: July 18, 2016

Published: July 18, 2016 


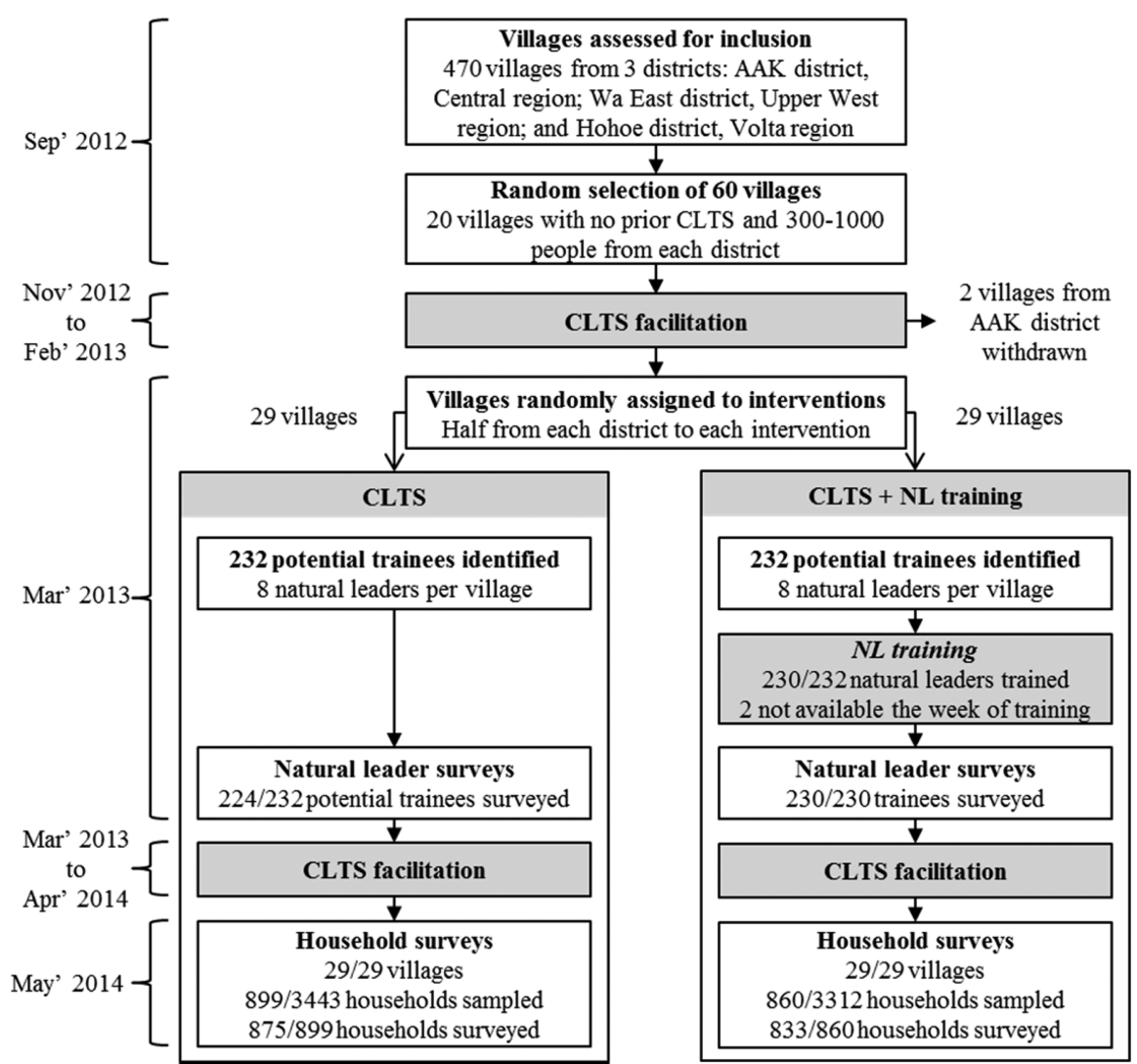

Figure 1. Timeline and sequence of the cluster-randomized field trial study design and execution. Two villages from the Central region were withdrawn from the study in December 2012 when village leaders turned down the intervention. Two natural leaders selected for training in the CLTS + NL training intervention were not trained, as they were not available during the week of training.

opinion leaders, in that they adopt a behavior (latrine use) quickly, influence their peers to do the same, and are not necessarily in positions of authority. Natural leaders have not been studied in any peer-reviewed literature.

Sanitation and hygiene behavior change interventions often incorporate recruitment and training of local actors to influence the behaviors of others. There are a number of evaluations of sanitation and hygiene interventions that have trained local actors, such as village leaders, ${ }^{13}$ health workers, ${ }^{8,13}$ teachers and students, ${ }^{8,14,15}$ and volunteer mothers. ${ }^{16}$ Each of these evaluations report positive behavioral outcomes. However, none could attribute outcomes to training, because training was always embedded within a broader project. Additionally, in each evaluation, trainees were identified by positions they held, rather than by their motivation or influence on peers, so they cannot necessarily be considered early adopters or opinion leaders. Trainee identification methods matter, because they can influence training effectiveness. Identifying trainees by position is quick and straightforward, but may identify trainees who are not motivated or influential. ${ }^{17} \mathrm{~A}$ cross-sectional study in India found that individuals were more influenced to adopt latrines by their peers than by their village as a whole, and suggested that socially relevant (influential) community members could be trained to influence the behavior of their peers. ${ }^{18}$ Trained opinion leaders have been shown to impact their peers' behaviors in other fields, for example on the quality of care provided by doctors, ${ }^{19}$ and on HIV-prevention behaviors among gay communities. ${ }^{20}$

The nongovernmental organization (NGO) Plan International Ghana (Plan) has worked on CLTS in Ghana since 2009. They have found that intensive, long-term follow-up is required, and there is frequently low community response. Plan proposed training natural leaders to build local capacity, improve community dynamics, and improve CLTS outcomes. We used a cluster-randomized field trial to evaluate the impact of training natural leaders as an add-on to CLTS. The primary outcome was sanitation practice as a categorical variable comprising open defecation, and communal, shared, and private latrine use. Secondary analysis focused on latrine quality, the implementation processes, and natural leader and community member activities. Multiple sites (regions in Ghana) were included to study how outcomes varied. This study was collaborative operational research to generate implications for policy and practice, with the University of North Carolina (UNC) leading the research and Plan leading implementation.

\section{MATERIALS AND METHODS}

Program Description. Two different interventions were implemented. The first ("CLTS") comprised the standard three stages of facilitation described in the Handbook on CLTS: ${ }^{4}$ pretriggering, triggering, and follow-up. The second ("CLTS + NL training") was standard CLTS with additional training given to natural leaders.

For both interventions, CLTS facilitation began in November 2012, and continued for 18 months. CLTS was facilitated by Plan and three local NGOs (one each in the Central, Upper West, and Volta regions). From here on, Plan and the local NGOs they contracted are referred to collectively as Plan. Plan identified eight natural leaders in each village from those that had emerged after 5 months of CLTS facilitation (pretrigging, triggering, and at least one follow-up visit). Identified natural leaders had built a 
latrine (or owned one) in the first 3-months of CLTS, consistently attended community meetings, and worked to convince others from their village to build a latrine. Plan ensured that at least one female per village was identified. Plan and district government officials trained the identified natural leaders in groups in regional capitals from half of the villages (the 'CLTS + NL training' intervention). Training comprised an initial 4-day session in March 2013, and three 1-day review meetings and a 4day refresher training over the following year. Training covered CLTS concepts, sanitation, handwashing, health outcomes, latrine construction, and participatory techniques. Training incorporated group work, simulations, and workplanning. It is important to note that natural leaders emerge regardless of this training, and external facilitators give them some guidance when visiting their villages. The additional training we evaluated comprised a more intensive training and took place outside their villages. The full training manual is available online. ${ }^{21}$ A timeline is in the supplement, and an implementation narrative by Plan is online. $^{22}$

The following pre-existing factors enabled the project. Plan had prior experience implementing CLTS, working with natural leaders, and collaborating with the government on sanitation and hygiene. The Government of Ghana had included CLTS in national policy, and established institutional support mechanisms, such coordinating committees at national and regional levels. $^{23}$

Study Design. A cluster-randomized field trial was used to evaluate training natural leaders as an addition to a CLTS intervention in Ghana, in comparison to CLTS without natural leader training. The primary author assigned half of the villages in each region to receive natural leader training using the random number function in Excel (Figure 1). Random assignment occurred after 5 months of facilitation, just before the initial NL training.

Sampling. The Central, Upper West, and Volta regions in Ghana were selected for inclusion in this project as they have different environmental and social characteristics, had high levels of OD, and Plan had an established relationship with regional government. One district was selected from each region in which the local government was familiar with CLTS and most villages had not received a CLTS intervention. Twenty villages with no prior CLTS, and a population of 300-1000 according to district records were randomly selected from each district. Two villages in the Central region withdrew from the study when village leaders turned down the intervention.

A complete household listing was completed in May 2014 in all study villages, and GPS data and number of people living in each household were recorded. Households were randomly sampled proportional to village population, with an 18household per village minimum. Twenty-six percent of all households were sampled (1759), of which 97\% were surveyed (1708). This sample size allowed for a detectable difference in open defecation between treatment groups ranging from 12 percentage points to 18 percentage points with $80 \%$ power, $95 \%$ significance level, and a conservative intracluster correlation of 0.2. ${ }^{7,24-26}$ Sampling details are in the supplement.

Data Collection. Demographics and sanitation and hygiene outcomes were measured using household surveys and surveyor observations in May 2014, just after the CLTS interventions ended. Indicators were selected based on prior $\mathrm{WaSH}$ research, ${ }^{12,26-28}$ and input from UNC and Plan. Surveys were administered by an independent contractor with experience in Ghana, one team-lead per region, and local surveyors. Surveys were translated into the local languages (Fante, Ewe, and Waale) by surveyors during training, translations checked for accuracy by team-leads, and then CLTS-related terms checked by Plan staff. The survey tools were developed in SurveyCTO software, and administered from Nexus tablets. Surveys were pretested and piloted during training. Team-leads reviewed survey responses and counts each evening, and audited surveyors by revisiting a random selection of households to verify data accuracy. Plan staff helped locate missed households.

Printed surveys on training content and CLTS-related knowledge, attitudes, and practices were administered to natural leaders in all 58 villages in April 2013 ( 1 month after the initial training). Natural leader and household surveys were administered by the same contractor and team.

Interviews with Plan and district government were used to understand the context, implementation process, and challenges. Government officials were interviewed in June 2012, before the interventions began. Plan staff were interviewed in December 2012 and March 2014, at the beginning and end of the interventions. Interviews were administered in English, which was comfortably spoken by all interviewees. The CLTS process was monitored with field checklists filled out by Plan, and questions in natural leader and household surveys.

Analysis. The primary outcome was household-level sanitation practice, as a categorical variable comprising (1) OD, and use of a (2) communal latrine, (3) shared latrine, or (4) private latrine. Self-reported latrine use was validated by observing latrines. Full, collapsed, and unstable latrines were categorized as OD. The impact of training natural leaders was evaluated using an ordered logistic regression model, because there are benefits associated with each step between open defecation and private latrine use $\mathrm{e}^{29}$ and all four practices were common among the study population. Change in latrine ownership over time was assessed based on respondents recalling how long they had owned their latrine. Recalled latrine age was paired with latrine observations to compare the quality of preexisting latrines to those built during CLTS. Analysis was completed in STATA 12/13/SE. Clustering of outcomes within villages, unequal selection probabilities, and nonresponse rates were accounted for using the "svyset" command.

This study was reviewed and approved by the Office of Human Research Ethics of the University of North Carolina, Chapel Hill (study \#12-1970). Local approval was obtained from regional environmental health and sanitation directorates in Ghana. Informed consent was received from all respondents.

\section{RESULTS}

Descriptive Analysis. Table 1 includes characteristics of households and respondents, by treatment group, from the follow-up survey. Variables that were not likely influenced by the interventions are presented, as they can be used to assess balance across treatment groups with postintervention surveys. Most characteristics are balanced; however, families in the CLTS + NL training treatment group had lived in their village an average of 5 years less than families in the CLTS treatment group. Villages were randomly assigned to ensure internal validity.

Villages in the Upper West region had different social characteristics than those in the Central and Volta regions (Table 2). There were fewer households in each village in the Upper West, fewer households in each compound, and more people living in each household. Families had lived in their villages longer. Fewer villages had prior externally funded $\mathrm{WaSH}$ projects and fewer households had received latrine hardware 
Table 1. Household and Respondent Characteristics From 29 Villages Receiving CLTS, and 29 Villages Receiving CLTS with Natural Leader Training Added On ${ }^{a}$

\begin{tabular}{|c|c|c|c|}
\hline variable & CLTS & $\begin{array}{l}\text { CLTS + NL } \\
\text { training }\end{array}$ & $\begin{array}{l}\text { difference } \\
(95 \% \mathrm{CI})\end{array}$ \\
\hline average village size (HHs) & 209 & 162 & $\begin{array}{l}-47(-153 \\
59)\end{array}$ \\
\hline average compound size & 2.3 & 2.7 & $0.3(-0.2,0.9)$ \\
\hline average household size & 4.1 & 3.9 & $-0.2(-1,0.5)$ \\
\hline children under five years of age & 0.7 & 0.6 & $\begin{array}{l}-0.1(-0.3 \\
0.1)\end{array}$ \\
\hline female respondent & $74 \%$ & $69 \%$ & $\begin{array}{l}-5 \%(-13 \% \\
2 \%)\end{array}$ \\
\hline average age & 44 & 43 & $-1(-4,3)$ \\
\hline completed primary school & $52 \%$ & $58 \%$ & $\begin{array}{c}7 \%(-8 \% \\
22 \%)\end{array}$ \\
\hline years family lived in village & 31 & 25 & $\begin{array}{l}-5(-10 \\
-0.3)\end{array}$ \\
\hline years family lived in current house & 15 & 14 & $-2(-5,1)$ \\
\hline metal/fabricated roofing & $88 \%$ & $93 \%$ & $\begin{array}{l}5 \%(-4 \% \\
14 \%)\end{array}$ \\
\hline TV ownership & $34 \%$ & $41 \%$ & $\begin{array}{c}7 \%(-3 \% \\
16 \%)\end{array}$ \\
\hline Radio ownership & $48 \%$ & $50 \%$ & $\begin{array}{c}2 \%(-6 \% \\
9 \%)\end{array}$ \\
\hline Cell phone owners/house & 1.2 & 1.4 & $0.2(-0.1,0.4)$ \\
\hline Use an improved water supply & $77 \%$ & $77 \%$ & $\begin{array}{l}0 \%(-10 \% \\
11 \%)\end{array}$ \\
\hline $\begin{array}{l}\text { Main water source is in dwelling or } \\
\text { compound }\end{array}$ & $9.0 \%$ & $10.9 \%$ & $\begin{array}{l}2 \%(-5 \% \\
9 \%)\end{array}$ \\
\hline Baseline private latrine ownership $^{b}$ & $9.4 \%$ & $12.7 \%$ & $\begin{array}{l}3 \%(-1 \%, \\
8 \%)\end{array}$ \\
\hline
\end{tabular}

${ }^{a_{T}}$ Twenty-nine villages received each intervention. Confidence intervals were generated with a $t$ test. ${ }^{b} \mathrm{~A}$ compound is a group of households arranged around a courtyard, often comprising an extended family. ${ }^{c}$ All values are taken from the 1.5-year follow up household census and survey, and describe the two treatment groups at that time, except for baseline private latrine ownership, which is based on recall of how old their latrines were.

Table 2. Characteristics of Study Population Receiving CLTS Interventions, by Region

\begin{tabular}{llll}
\multicolumn{1}{c}{ variable } & central & upper west & volta \\
\hline village size (number of households) & 164 & 67 & 122 \\
compound size (number of households) & 2.5 & 1.8 & 2.8 \\
household size (number of people) & 3.3 & 6.4 & 3.6 \\
number of children per household $^{\text {average family tenure (years in village) }}$ & 0.5 & 1.1 & 0.5 \\
population density (people/sq. km) $^{a}$ & 27 & 36 & 25 \\
prior WaSH project (\% of villages) $^{b}$ & $100 \%$ & $45 \%$ & $79 \%$ \\
prior HH latrine subsidy (\% of villages) $^{b}$ & $33 \%$ & $15 \%$ & $37 \%$
\end{tabular}

${ }^{a}$ Based on GPS data from the household census conducted for the follow-up survey. ${ }^{b}$ From situational assessments conducted in 2012 before implementation began. ${ }^{23}$

subsidies. The villages in the Upper West and Volta regions had lower population densities than those in the Central region.

Sanitation Outcomes. Training natural leaders as an addition to CLTS caused a 19.9 percentage point (pp) reduction in households practicing OD, as compared to villages that just received CLTS (95\% CI: -8.8 to $-30.9 \mathrm{pp}, p<0.001$ ) (Figure 2 ). The impact was largest in villages in the Upper West region, at a $38.6 \mathrm{pp}$ reduction in OD ( $95 \% \mathrm{CI}$ : -14.2 to $-63.0 \mathrm{pp}, \mathrm{p}=$ 0.002 ). There was a smaller reduction in OD in the villages in the
Volta region ( $-12.4 \mathrm{pp}, 95 \% \mathrm{CI}:-5.1$ to $-19.7 \mathrm{pp}, \mathrm{p}=0.001)$, and no statistically significant impact in the Central region.

In the full sample, the reduction in OD caused by training natural leaders corresponded to a small increase in shared latrine use (4.3 pp, 95\% CI: 1.7 to $6.9 \mathrm{pp}$ ), and a larger increase in private latrine use (18.3 pp, 95\% CI: 9.5 to $27.1 \mathrm{pp}$ ) (Figure 3). Communal latrine use was common in both treatment groups in the Central and Volta regions, but not practiced by any households in the Upper West region (data in the supplement).

Table 3 shows household private latrine ownership by region and treatment group. Ownership (Table 3 ) is differentiated from use of a private latrine (Figure 3), as use includes rented latrines (families renting a house with a latrine). There was an increase in the percentage of households owning a private latrine across regions and treatment groups. The largest increases in private latrine ownership occurred in the Upper West and Volta regions and, within those regions, in the CLTS + NL training group.

Latrines built during both the CLTS and CLTS + NL training interventions were on average less durable than pre-existing latrines. A greater proportion of pre-existing latrines had ventilation pipes and intact superstructures that offered privacy and protection from the weather (Table 4). Pre-existing latrines also more often qualified as "improved latrines". However, latrines built during CLTS and pre-existing latrines were comparably clean and had similar fly levels. A greater proportion of latrines built during CLTS had handwashing materials available. Latrines in villages in the Central region were of higher quality than latrines in the Upper West and Volta regions (data in the Supporting Information). The more densely populated Central region has better access to markets. Latrines were observed after the CLTS interventions, so owners of preexisting latrines may have upgraded or maintained them during the CLTS interventions. There were no significant differences in quality between new latrines built in the CLTS and CLTS + NL training villages (data not presented).

Implementation Process. During the 1.5 year implementation period, Plan facilitators averaged 12.1 visits per village that received just CLTS, and 12.9 visits per village that received CLTS $+\mathrm{NL}$ training (Table 5). The 0.8 -visit difference was for a visit to invite natural leaders to the initial training. Community participation in triggering (which occurred before NL training) was similar across treatment groups, though it varied between regions. After triggering was completed in all villages, eight natural leaders were trained per village in the CLTS + NL training half of the villages. This represents a larger proportion of the smaller Upper West villages.

At the follow-up survey, the percentage of community members participating in sanitation and hygiene-related activities and interactions was nearly identical across treatment groups (Table 6). For both treatment groups, approximately one-third of community members reported attending any sanitation or hygiene meeting in the past two months, and approximately onethird reported discussing sanitation or hygiene with a neighbor over the same period. However, the intensity (total level of activity) was higher in villages where natural leaders had been trained. Time spent promoting sanitation and hygiene within villages by natural leaders and government per 10000 people targeted was approximately $130 \%$ higher in villages where natural leaders were trained, and community time on CLTS was $80 \%$ higher (excludes time in training) (Table 6). 


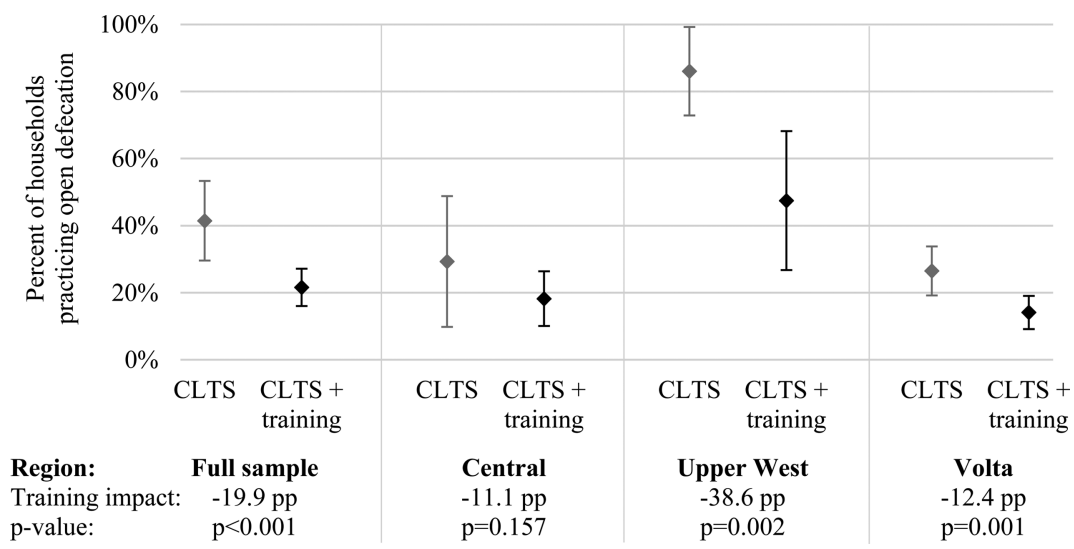

Figure 2. The full sample is 58 villages: 18 in Central, 20 in Upper West, and 20 in Volta, split evenly between interventions. Diamond-shaped points are means, and bars are $95 \%$ confidence intervals. Open defecation is modeled from ordered logistic regression parameters. Analysis accounts for unequal selection probability, nonresponse rates, and village clustering. ICC $=0.618$ for $\mathrm{OD}$ at the village level in the full sample. Impact of training natural leaders on open defecation as an addition to CLTS in Ghana, full sample and by region.

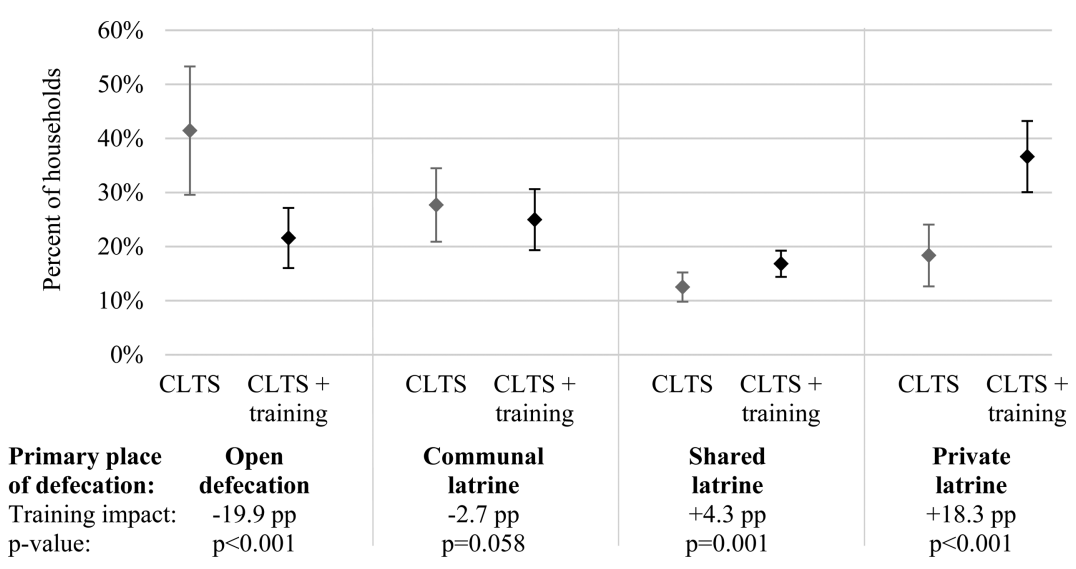

Figure 3. Fifty-eight villages were included in the evaluation, split evenly between interventions. Diamond-shaped points are means, and bars are $95 \%$ confidence intervals. Sanitation practice is modeled from ordered logistic regression parameters (in the supplement). Estimates and standard errors account for unequal selection probability, nonresponse rates, and village clustering. Impact on sanitation practice of training natural leaders as an addition to CLTS in Ghana.

\section{DISCUSSION}

The impact of adding natural leader training to a CLTS intervention in Ghana was a 19.9 percentage point (pp) reduction in OD. This should be interpreted as the impact of training natural leaders, not of natural leaders in general, as natural leaders emerged in all project villages. Villages were enrolled from three dissimilar regions in order to study how the impact varied between different settings. The impact was far larger in villages in the Upper West $(-38.6 \mathrm{pp})$, than in the Central and Volta regions $(-12.4$ and $-11.1 \mathrm{pp})$. The reduction in OD corresponded to a large impact on private latrine use (+18.3 pp), and little to no impact on shared or communal latrine use. This evaluation took place in the presence of a strong enabling environment, ${ }^{8}$ which is common as CLTS is incorporated into policy in many countries and is supported by many organizations. ${ }^{32}$

Latrines built during CLTS were, on average, less likely to be made of durable materials or to have a fully intact superstructure compared to pre-existing latrines. However, pre-existing latrines and those built during CLTS were comparable regarding cleanliness, use of hole covers, and presence of flies, indicating they were similarly maintained and protected users from exposure to fecal matter. Latrines built during CLTS were more likely to have handwashing materials present, indicating more attention to hygiene, which is important given latrine access can associate with fecal contamination of hands. ${ }^{33}$ CLTS focuses on behavior and does not prescribe latrine types, which others have found mean cheap, nondurable latrines are built. ${ }^{8,34,35}$ Still, the majority of latrines built during this study were durable and stable. We reported latrine characteristics in addition to the "improved" indicator, as improved latrines are not necessarily safe. $^{36}$

Plan conducted similar intensities of facilitation in both treatment groups to ensure that any differences in outcomes could be attributed to training natural leaders. At triggering, community participation was similar across treatment groups, indicating that it did not diverge before natural leaders were trained. Just after the interventions, the percentage of households participating in sanitation and hygiene meetings and discussions was still equal across treatment groups, indicating that training natural leaders did not increase the proportion of their villages reached by the CLTS message. However, sanitation and hygienerelated activity levels and interaction was much higher in villages where natural leaders were trained. Natural leaders spent more time reinforcing CLTS messages, and community members spent more in meetings, discussions, and building latrines.

A small number of trained individuals (less than two natural leaders were trained per 100 community members) influenced 
Table 3. Household Ownership of a Private Latrine before and after CLTS and CLTS + NL Training in Ghana, by Region and Intervention $^{a}$

\begin{tabular}{|c|c|c|c|c|c|}
\hline \multirow[b]{2}{*}{ region } & \multirow[b]{2}{*}{ treatment } & \multicolumn{2}{|c|}{$\begin{array}{l}\text { private latrine } \\
\text { ownership }^{b}\end{array}$} & \multirow[b]{2}{*}{ change } & \multirow[b]{2}{*}{$p$-value } \\
\hline & & $\begin{array}{c}\text { pre- } \\
\text { CLTS }^{c}\end{array}$ & $\begin{array}{l}\text { post- } \\
\text { CLTS }\end{array}$ & & \\
\hline \multirow[t]{2}{*}{ central } & CLTS & $11.6 \%$ & $15.7 \%$ & $4.1 \mathrm{pp}$ & 0.005 \\
\hline & $\begin{array}{l}\text { CLTS + NL } \\
\text { training }\end{array}$ & $14.6 \%$ & $21.6 \%$ & $7.0 \mathrm{pp}$ & 0.001 \\
\hline \multirow[t]{2}{*}{ upper west } & CLTS & $5.0 \%$ & $14.5 \%$ & $9.6 \mathrm{pp}$ & 0.075 \\
\hline & $\begin{array}{l}\text { CLTS + NL } \\
\text { training }\end{array}$ & $13.9 \%$ & $52.3 \%$ & $38.4 \mathrm{pp}$ & 0.001 \\
\hline \multirow[t]{2}{*}{ volta } & CLTS & $9.7 \%$ & $18.9 \%$ & $9.2 \mathrm{pp}$ & 0.013 \\
\hline & $\begin{array}{c}\text { CLTS }+\mathrm{NL} \\
\text { training }\end{array}$ & $9.9 \%$ & $23.9 \%$ & $13.9 \mathrm{pp}$ & 0.004 \\
\hline
\end{tabular}

${ }^{a_{T}}$ The study included 18 villages in Central, 20 in Upper West, and 20 in Volta, split evenly between interventions in each region. Percentages account for unequal selection probability and nonresponse rates. ICC $=0.349$ for ownership of a usable latrine at the village level in the full sample. P-values were generated with a $t$ test. ${ }^{b}$ Private latrine ownership is different from use in Figure 3, as use includes families renting houses with latrines. ${ }^{c}$ Based on respondent recall of the age of their latrine at the follow-up survey.

the collective sanitation behavior of their communities without money or latrine materials being provided to them or their villages. While no prior studies demonstrate an impact of training community members on $\mathrm{WaSH}$ behaviors, these findings seem plausible considering prior studies found that social network interactions predict latrine adoption, ${ }^{18}$ and that latrine adoption decisions among community members are interlinked and spur more latrine adoption. $7,37,38$

Trained natural leaders were most able to influence behaviors in villages with indicators of higher social cohesion, in the Upper West region where villages were smaller, more remote, and families had lived in the villages longer. These same villages had lower exposure to externally supported $\mathrm{WaSH}$ projects. This aligns with assertions in the CLTS Handbook, ${ }^{4}$ that, where prior latrine subsidies occurred, expectation of external support can hinder collective action. This also aligns with empirical evidence from a study in Tanzania that found social capital influences CLTS effectiveness. ${ }^{9}$ The better outcomes in the Upper West region could also be explained by the higher proportion of each village trained (eight natural leaders were trained per village, and villages in the Upper West were smaller). Variation in outcomes is expected for interventions targeting environmental health behaviors, as both behaviors and exposure are influenced by social and environmental factors.

The effectiveness of the natural leader training at increasing interactions and impacting behaviors fits with diffusion theory, in which adoption is initiated by external factors (e.g., triggering), while diffusion beyond early adopters is supported by endogenous factors, such as peer-communication and opinion leaders (e.g., natural leaders). ${ }^{11}$ Plan waited to identify natural leaders to train in Ghana until after triggering when natural leaders had multiple opportunities to demonstrate their motivation by constructing latrines and influencing their peers. This method has the advantage of identifying trainees who are likely motivated and influential. ${ }^{17}$ It is also unique from prior sanitation and hygiene studies, which have trained local actors that can be identified at the outset of the project (e.g., village leaders, health workers, and teachers). Interventions that focus on training actors easily identified at the outset could be failing due to not training socially relevant individuals. ${ }^{18}$ By targeting individuals based on their title or profession, prior interventions are assuming that high visibility individuals in positions of authority are motivated and influential. The CLTS concept of natural leaders, and the impact that training them had in Ghana, provides direct evidence of the potential of opinion leaders to influence sanitation and hygiene behaviors within their community.

Our study provides new evidence that training natural leaders in participatory, social, and technical skills during a behavior change intervention can substantially reduce open defecation in rural villages. Adding natural leader (or opinion leader) training to other environmental health behavior change programs should

Table 4. Characteristics of Latrines Built During CLTS Compared to Pre-Existing Latrines in 58 Villages in Ghana ${ }^{a}$

\begin{tabular}{|c|c|c|c|c|c|}
\hline & \multirow[b]{2}{*}{ variable } & \multicolumn{2}{|c|}{ latrine built } & \multirow[b]{2}{*}{$p$-value } & \multirow[b]{2}{*}{ CLTS latrines compared to pre-existing latrines } \\
\hline & & pre-CLTS & during CLTS & & \\
\hline \multirow[t]{8}{*}{ infrastructure } & durable flooring material ${ }^{b}$ & $84 \%$ & $60 \%$ & $<0.001$ & less durable flooring \\
\hline & stable/safe flooring & $94 \%$ & $86 \%$ & 0.005 & slightly less stable/safe flooring \\
\hline & fully intact walls & $71 \%$ & $55 \%$ & 0.002 & less likely to have intact walls \\
\hline & intact door & $77 \%$ & $47 \%$ & $<0.001$ & less likely to have an intact door \\
\hline & protective roof & $79 \%$ & $57 \%$ & $<0.001$ & less likely to have an intact roof \\
\hline & pit ventilation & $56 \%$ & $31 \%$ & $<0.001$ & less likely to be ventilated \\
\hline & complete privacy & $66 \%$ & $48 \%$ & 0.003 & less privacy provided \\
\hline & improved latrine $^{c}$ & $52 \%$ & $43 \%$ & 0.026 & less likely to be "improved" \\
\hline \multirow[t]{4}{*}{ upkeep } & hole covered & $50 \%$ & $47 \%$ & 0.584 & comparable hole coverage \\
\hline & clean (no feces on floor) & $83 \%$ & $83 \%$ & 0.869 & comparable cleanliness \\
\hline & less than $\sim 10$ flies & $74 \%$ & $70 \%$ & 0.316 & comparable level of flies \\
\hline & water or cleansing material for handwashing & $6 \%$ & $21 \%$ & $<0.001$ & better access to handwashing facilities \\
\hline
\end{tabular}

${ }^{a}$ Analysis covers the 530 of 554 privately owned latrines and 213 of 264 shared latrines that were observed during surveying in villages in both the CLTS and CLTS + NL Training interventions. Latrines placed in the pre-CLTS category were reported as more than 18 months old. Pre-CLTS includes 447 latrines, and during CLTS includes 296 latrines. Analysis accounts for unequal selection probability, nonresponse rates, and village clustering. P-values were generated with a $t$ test. ${ }^{b}$ Concrete or wood. ${ }^{c}$ Based on the Joint Monitoring Program definition, though measurement of improved latrines varies globally. ${ }^{30,31}$ 
Table 5. Implementation Details-Village Visits, Community Presence at Triggering, and Natural Leaders Characteristics, by Region and Treatment Group ${ }^{a}$

\begin{tabular}{|c|c|c|c|c|c|c|}
\hline treatment & region & $\begin{array}{l}\text { follow-up visits } \\
\text { per village }\end{array}$ & $\begin{array}{l}\text { community hours in CLTS triggering, per } \\
10,000 \text { ppl targeted }\end{array}$ & $\begin{array}{l}\text { average age of } \\
\text { identified NLs }\end{array}$ & $\begin{array}{l}\text { percent of identified NLs } \\
\text { that were female }\end{array}$ & $\begin{array}{l}\text { NLs trained per } \\
\text { village }\end{array}$ \\
\hline \multirow[t]{4}{*}{ CLTS } & Central & 12.6 & 7360 & 47 & $21 \%$ & 0 \\
\hline & $\begin{array}{l}\text { Upper } \\
\text { West }\end{array}$ & 10.5 & 11187 & 42 & $27 \%$ & 0 \\
\hline & Volta & 13.2 & 9752 & 50 & $29 \%$ & 0 \\
\hline & average & 12.1 & 9424 & 46 & $26 \%$ & 0 \\
\hline \multirow{4}{*}{$\begin{array}{l}\text { CLTS + NL } \\
\text { training }\end{array}$} & Central & 13.1 & 8634 & 43 & $33 \%$ & 8.0 \\
\hline & $\begin{array}{l}\text { Upper } \\
\text { West }\end{array}$ & 11.5 & 8295 & 35 & $27 \%$ & 7.8 \\
\hline & Volta & 14.2 & 9383 & 47 & $37 \%$ & 8.0 \\
\hline & average & 12.9 & 8814 & 42 & $32 \%$ & 7.9 \\
\hline
\end{tabular}

a "NL" = natural leader. Each treatment group included 29 villages. Natural leaders were present in all villages, but were only trained in the CLTS + NL training group. ${ }^{b}$ Follow-up visits includes visits by Plan, local NGOs, and government. ${ }^{c}$ Triggering occurred between December $2012-$ March 2013, before natural leader training. Villages were randomly assigned to treatment groups after triggering, so any difference in community hours in triggering is due to chance. Hours in triggering were measured using checklists to record attendance and duration of each triggering. ${ }^{d}$ Natural leader training occurred in March 2013, after triggering was complete in all villages.

Table 6. Implementation Details-local Actor and Community Activity During CLTS, by Region and Treatment

\begin{tabular}{|c|c|c|c|c|c|}
\hline treatment & region & $\begin{array}{l}\text { attended village sanitation/hygiene } \\
\text { meeting in past } 2 \text { months }\end{array}$ & $\begin{array}{c}\text { discussed sanitation/hygiene with a } \\
\text { neighbor in past } 2 \text { months }\end{array}$ & $\begin{array}{l}\text { local actor hours on CLTS, } \\
\text { per } 10000 \text { people }^{a}\end{array}$ & $\begin{array}{c}\text { community hours on CLTS, } \\
\text { per } 10000 \text { people }^{b}\end{array}$ \\
\hline \multirow[t]{4}{*}{ CLTS } & Central & $30 \%$ & $29 \%$ & 707 & 18084 \\
\hline & $\begin{array}{l}\text { Upper } \\
\text { West }\end{array}$ & $29 \%$ & $30 \%$ & 1368 & 23838 \\
\hline & Volta & $43 \%$ & $52 \%$ & 3233 & 44569 \\
\hline & Average & $30 \%$ & $34 \%$ & 1696 & 28037 \\
\hline \multirow{4}{*}{$\begin{array}{l}\text { CLTS + NL } \\
\text { training }\end{array}$} & Central & $40 \%$ & $54 \%$ & 2423 & 44017 \\
\hline & $\begin{array}{l}\text { Upper } \\
\text { West }\end{array}$ & $20 \%$ & $29 \%$ & 4319 & 49730 \\
\hline & Volta & $28 \%$ & $33 \%$ & 5102 & 53982 \\
\hline & Average & $30 \%$ & $35 \%$ & 3925 & 49236 \\
\hline
\end{tabular}

${ }^{a}$ Includes time spent by natural leaders and government promoting sanitation and hygiene within study villages, and excludes travel and training time. Hours on CLTS were measured using checklists to track attendance at CLTS meetings, and surveys to estimate time in CLTS outside of meetings. ${ }^{b}$ Includes time in CLTS meetings and visits, as well as time spent on latrine construction.

be considered, as should targeting training to socially cohesive communities where it is likely to have the largest impact. Scheduling training after initial triggering and some follow-up has occurred enables selection of trainees who demonstrate they are motivated by communal outcomes and work to influence peers, and enables filtering out potential trainees who are seeking personal gain such as financial benefits. CLTS should not be a standalone strategy for addressing sanitation, given that some of the resulting latrines were low quality, and some regions saw minimal increase in latrine coverage. With inclusion of natural leader training targeted to appropriate villages, CLTS can play a role in addressing three parts of Sustainable Development Goal (SDG) 6: eliminating OD, expanding capacity-building in developing countries, and strengthening community participation. ${ }^{39}$

Our findings were enabled by a study design suited to operational research in WaSH. There have been many randomized trials in $\mathrm{WaSH}$; all compare an intervention to a "do nothing" control group. ${ }^{25,40-42}$ This is the first WaSH evaluation in which a modification or addition to an intervention is compared to an existing approach within a randomized trial. Situational assessments, implementation tracking, and survey questions on sanitation-related activities supported assessing the implementation process and mechanism by which the training impacted sanitation outcomes. A multisite study design enabled disaggregation of outcomes, and informs targeting of CLTS to appropriate settings, which is critical given there is no "one-size fits all" sanitation and hygiene intervention. ${ }^{43}$ To meet the SDG Targets for $\mathrm{WaSH}$, more evidence is needed on how to adapt and target interventions to a wide range of settings. Evaluations that investigate modifications to interventions, the implementation process, and how outcomes vary between settings can provide this much-needed evidence.

Limitations. This study has several potential limitations. It did not include a baseline survey, so baseline descriptive statistics and changes in sanitation practices over time could not be assessed. Variables measured in the postintervention survey that are slow to change and not likely influenced by CLTS were instead used to describe the comparison groups. Three recent CLTS evaluations have used baseline surveys to assess sanitation changes over time, ${ }^{5,7,8}$ so this study instead focused on the impact of training natural leaders. The protocol for this study was reviewed by the project team, and reviewed and approved by the UNC Office of Human Research Ethics; however, the protocol was not posted to an open-access site such as clinicaltrials.gov. Each of the three regions in this study had different facilitators and trainers, which could have caused some of the variation in outcomes between regions. To minimize this, the facilitators and 
trainers agreed on implementation details at the project outset. Implementation was monitored, reviewed monthly, and adjusted when it differed between regions. Plan's project manager attended all training sessions in each region to ensure consistency.

\section{ASSOCIATED CONTENT}

\section{(5 Supporting Information}

The Supporting Information is available free of charge on the ACS Publications website at DOI: 10.1021/acs.est.6b01557.

Details on implementation, sampling, outcomes, regressions, and the CONSORT checklist. ${ }^{44}$ (PDF)

\section{AUTHOR INFORMATION}

\section{Corresponding Author}

*Phone: (919) 843-3393; e-mail: jonny.crocker@unc.edu.

\section{Notes}

The authors declare the following competing financial interest(s): Elvis Abodoo, Daniel Asamani, William Domapielle, and Benedict Gyapong are involved in ongoing CLTS implementation in Ghana.

\section{ACKNOWLEDGMENTS}

This research is a result of a subagreement to the University of North Carolina at Chapel Hill from Plan International USA, which received a grant from the Bill \& Melinda Gates Foundation (OPP1028953). A National Institute of Environmental Health Sciences Training Grant (T32ES007018) supported the primary author. Neither funder had any role in study design, execution, or article preparation. We thank the study participants; Plan International USA for grant management; Plan International Ghana for implementation; Elana Cohen-Khani for administering surveys; Kate Shields for data cleaning; and Chris Wiesen at the Odum Institute at UNC for supporting analysis.

\section{REFERENCES}

(1) WHO/UNICEF. Progress on Sanitation and Drinking Water: 2015 update and MDG Assessment; Geneva, Switzerland, 2015.

(2) Prüss-Ustün, A.; Bartram, J.; Clasen, T. F.; Colford, J. M.; Cumming, O.; Curtis, V. A.; Bonjour, S.; Dangour, A. D.; De France, J.; Fewtrell, L.; et al. Burden of disease from inadequate water, sanitation and hygiene in low- and middle-income settings: a retrospective analysis of data from 145 countries. Trop. Med. Int. Health 2014, 19 (8), 894905.

(3) Evans, W. D.; Pattanayak, S. K.; Young, S.; Buszin, J.; Rai, S.; Bihm, J. W. Social marketing of water and sanitation products: a systematic review of peer-reviewed literature. Soc. Sci. Med. 2014, 110, 18-25.

(4) Kar, K.; Chambers, R. Handbook on Community-Led Total Sanitation; Plan UK and Institute of Development Studies, 2008; Vol. 44.

(5) Pickering, A. J.; Djebbari, H.; Lopez, C.; Coulibaly, M.; Alzua, M. L. Effect of a community-led sanitation intervention on child diarrhoea and child growth in rural Mali: a cluster-randomised controlled trial. Lancet Glob. Heal. 2015, 3, e701-e711.

(6) Briceno, B.; Coville, A.; Martinez, S. Promoting handwashing and sanitation: evidence from a large-scale randomized trial in rural Tanzania; Policy Research Working Paper; 7164; Washington, DC, 2015.

(7) Guiteras, R.; Levinsohn, J.; Mobarak, A. M. Encouraging sanitation investment in the developing world: a cluster-randomized trial. Science 2015, 348 (6237), 903-906.

(8) Crocker, J.; Geremew, A.; Atalie, F.; Yetie, M.; Bartram, J. Teachers and sanitation promotion: an assessment of community-led total sanitation in Ethiopia. Environ. Sci. Technol. 2016, 50, 6517-6525.
(9) Cameron, L.; Olivia, S.; Shah, M. Initial conditions matter: social capital and participatory development 2015, DOI: 10.2139/ssrn.2704614.

(10) Platteau, J.-P.; Abraham, A. Participatory development in the presence of endogenous community imperfections. J. Dev. Stud. 2002, 39 (2), 104-136.

(11) Rogers, E. M. Diffusion of innovations, 5th ed.; Free Press: New York, 2003.

(12) Venkataramanan, V. Testing CLTS approaches for scalability: systematic literature review. 2012. https://waterinstitute.unc.edu/files/ 2015/03/CLTS-Systematic-Literature-Review-Final-Report-Appendix1-4.pdf.

(13) Lee, W.; Stoeckel, J.; Jintaganont, P.; Romanarak, T.; Kullavanijaya, $S$. The impact of a community based health education program on the incidence of diarrheal disease in southern Thailand. Southeast Asian J. Trop. Med. Public Heal. 1991, 22 (4), 548-556.

(14) Blanton, E.; Ombeki, S.; Oluoch, G. O.; Mwaki, A.; Wannemuehler, K.; Quick, R. Evaluation of the role of school children in the promotion of point-of-use water treatment and handwashing in schools and households-Nyanza Province, Western Kenya, 2007. Am. J. Trop. Med. Hyg. 2010, 82 (4), 664-671.

(15) Onyango-Ouma, W.; Aagaard-Hansen, J.; Jensen, B. B. The potential of schoolchildren as health change agents in rural western Kenya. Soc. Sci. Med. 2005, 61, 1711-1722.

(16) Kariuki, J. G.; Magambo, K. J.; Njeruh, M. F.; Muchiri, E. M.; Nzioka, S. M.; Kariuki, S. Changing mother's hygiene and sanitation practices in resource constrained communities: case study of Turkana District, Kenya. J. Community Health 2012, 37 (6), 1178-1184.

(17) Valente, T. W.; Pumpuang, P. Identifying opinion leaders to promote behavior change. Heal. Educ. Behav. 2007, 34 (6), 881-896.

(18) Shakya, H. B.; Christakis, N. A.; Fowler, J. H. Association between social network communities and health behavior: an observational sociocentric network study of latrine ownership in rural India. Am. J. Public Health 2014, 104 (5), 930-937.

(19) Soumerai, S. B.; McLaughlin, T. J.; Gurwitz, J. H.; Guadanoli, E.; Hauptman, P. J.; Borbas, C.; Morris, N.; McLaughlin, B.; Gao, X.; Willison, D. J.; et al. Effect of local medical opinion leaders on quality of care for acute myocardial infarction: a randomized controlled trial. JAMA 1998, 279 (17), 1358-1363.

(20) Kelly, J. A.; Murphy, D. A.; Sikkema, K. J.; McAuliffe, T. L.; Roffman, R. A.; Solomon, L. J.; Winett, R. A.; Kalichman, S. C. Randomised, controlled, community-level HIV-prevention intervention for sexual-risk behaviour among homosexual men in US cities. Lancet $1997,350,1500-1505$.

(21) Plan International Ghana. Natural leaders training guide, 2013, 90 pp. http://scalingclts.web.unc.edu/files/2014/10/guide-ghana.pdf

(22) Plan International Ghana. CLTS with Capacity Building for Natural Leaders: Implementation Narrative; 2015. https://waterinstitute. unc.edu/files/2015/12/clts-gates-ghana-2015.pdf.

(23) Crocker, J.; Bogle, J. Community-Led Total Sanitation in Ghana: Findings from a Situational Assessment; Chapel Hill, NC, 2015. http:// waterinstitute.unc.edu/files/2015/03/situational-assessment-ghana2015-02.pdf.

(24) Freeman, M. C.; Clasen, T. F.; Brooker, S. J.; Akoko, D. O.; Rheingans, R. The impact of a school-based hygiene, water quality and sanitation intervention on soil-transmitted helminth reinfection: a cluster-randomized trial. Am. J. Trop. Med. Hyg. 2013, 89 (5), 875-883.

(25) Clasen, T. F.; Boisson, S.; Routray, P.; Torondel, B.; Bell, M.; Cumming, O.; Ensink, J.; Freeman, M. C.; Jenkins, M. W.; Odagiri, M.; et al. Effectiveness of a rural sanitation programme on diarrhoea, soiltransmitted helminth infection, and child malnutrition in Odisha, India: a cluster-randomised trial. Lancet Glob. Heal. 2014, 2 (14), e645-e653.

(26) Pattanayak, S. K.; Yang, J.-C.; Dickinson, K. L.; Poulos, C.; Patil, S. R.; Mallick, R. K.; Blitstein, J. L.; Praharaj, P. Shame or subsidy revisited: social mobilization for sanitation in Orissa, India. Bull. World Health Organ. 2009, 87 (8), 580-587.

(27) Hernandez, O.; Tobias, S. Access and behavioral outcome indicators for water, sanitation, and hygiene. USAID Hygiene Improvement Project 2010, 81. 
(28) Grosh, M.; Glewwe, P. Designing Household Survey Questionnaires for Developing Countries: Lessons from 15 Years of the Living Standards Measurement Study; The World Bank: Washington, DC, 2000.

(29) Heijnen, M.; Cumming, O.; Peletz, R.; Chan, G. K.-S.; Brown, J.; Baker, K. K.; Clasen, T. F. Shared sanitation versus individual household latrines: a systematic review of health outcomes. PLoS One 2014, 9 (4), $1-9$.

(30) Bartram, J.; Brocklehurst, C.; Fisher, M. B.; Luyendijk, R.; Hossain, R.; Wardlaw, T.; Gordon, B. Global monitoring of water supply and sanitation: history, methods and future challenges. Int. J. Environ. Res. Public Health 2014, 11, 8137-8165.

(31) WHO/UNICEF. Progress on Drinking Water and Sanitatation:

2014 Update; Geneva, Switzerland, 2014.

(32) Venkataramanan, V. CLTS Learning Series: Lessons From CLTS Implementation in Seven Countries; Chapel Hill, NC, 2016.

(33) Pickering, A. J.; Davis, J.; Walters, S. P.; Horak, H. M.; Keymer, D. P.; Mushi, D.; Strickfaden, R.; Chynoweth, J. S.; Liu, J.; Blum, A.; et al. Hands, water, and health: fecal contamination in Tanzanian communities with improved, non-networked water supplies. Environ. Sci. Technol. 2010, 44 (9), 3267-3272.

(34) Tyndale-Biscoe, P.; Bond, M.; Kidd, R. Plan International ODF Sustainability Study. 2013

(35) Whaley, L.; Webster, J. The effectiveness and sustainability of two demand-driven sanitation and hygiene approaches in Zimbabwe. $J$. Water, Sanit. Hyg. Dev. 2011, 1 (1), 20-36.

(36) Exley, J. L. R.; Liseka, B.; Cumming, O.; Ensink, J. H. J. The sanitation ladder, what constitutes an improved form of sanitation? Environ. Sci. Technol. 2015, 49 (2), 1086-1094.

(37) Dickinson, K.; Pattanayak, S. K. Open Sky Latrines: Social Reinforcing in the Case of a (Very) Impure Public Good. 2009

(38) Jenkins, M. W.; Cairncross, S. Modelling latrine diffusion in Benin: toward a community typology of demand for improved sanitation in developing countries. J. Water Health 2010, 8 (1), 166183.

(39) UN General Assembly. Draft Outcome Document of the United Nations Summit for the Adoption of the Post-2015 Development Agenda, 2015.

(40) Fewtrell, L.; Kaufmann, R. B.; Kay, D.; Enanoria, W.; Haller, L.; Colford, J. M., Jr Water, sanitation, and hygiene interventions to reduce diarrhoea in less developed countries: a systematic review and metaanalysis. Lancet Infect. Dis. 2005, 5, 42-52.

(41) Clasen, T. F.; Bostoen, K.; Schmidt, W.-P.; Boisson, S.; Fung, I. C. H.; Jenkins, M. W.; Scott, B.; Sugden, S.; Cairncross, S. Interventions to improve disposal of human excreta for preventing diarrhoea (review). Cochrane Libr. 2010, No. No. 6, 32.

(42) Waddington, H.; Snilstveit, B.; White, H.; Fewtrell, L. Water, Sanitation and Hygiene Interventions to Combat Childhood Diarrhoea in Developing Countries. 2009

(43) Tilley, E.; Strande, L.; Lüthi, C.; Mosler, H.-J.; Udert, K. M.; Gebauer, H.; Hering, J. G. Looking beyond technology: an integrated approach to water, sanitation and hygiene in low income countries. Environ. Sci. Technol. 2014, 48 (17), 9965-9970.

(44) Moher, D.; Schulz, K. F.; Altman, D. G. The CONSORT statement: revised recommendations for improving the quality of reports of parallel-group randomised trials. Lancet 2001, 357, 11911194. 